This item was submitted to Loughborough's Institutional Repository (https://dspace.lboro.ac.uk/) by the author and is made available under the following Creative Commons Licence conditions.

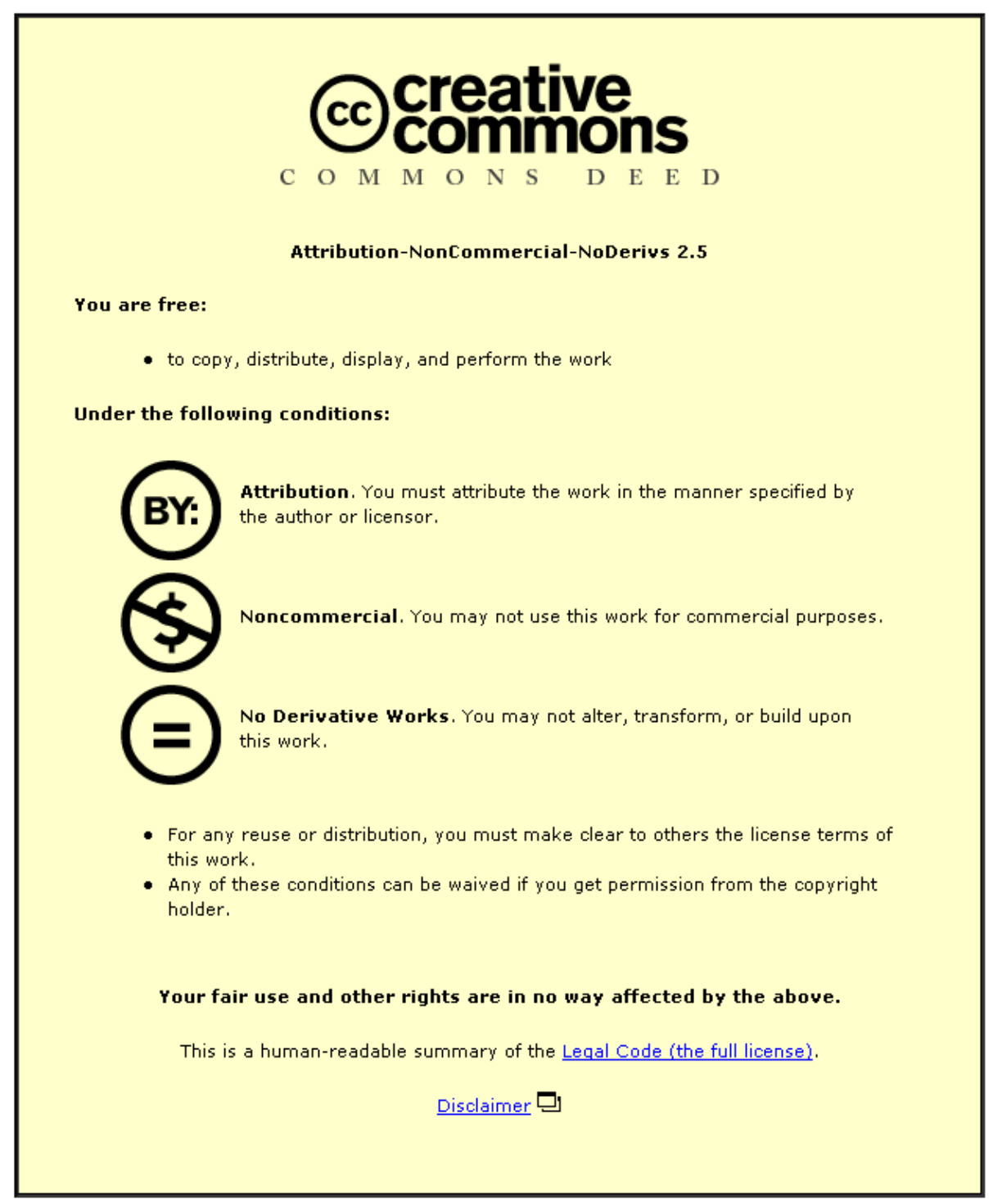

For the full text of this licence, please go to: http://creativecommons.org/licenses/by-nc-nd/2.5/ 
Journal of Biomechanics 46, 645-650, 2013.

\title{
The torque-velocity relationship in large human muscles: maximum voluntary versus electrically stimulated behaviour
}

\author{
${ }^{1}$ Matthew T.G. Pain, ${ }^{1}$ Fraser Young, ${ }^{1}$ Jinwoo Kim, and ${ }^{2}$ Stephanie E. Forrester \\ ${ }^{1}$ School of Sport, Exercise and Health Sciences, Loughborough University, Loughborough, \\ LE11 3TU, UK \\ ${ }^{2}$ Wolfson School of Mechanical and Manufacturing Engineering, Loughborough University, \\ Loughborough, LE11 3TU, UK
}

\begin{abstract}
The in vivo maximum voluntary torque-velocity profile for large muscle groups differs from the in vitro tetanic profile with lower than expected eccentric torques. Using sub-maximal transcutaneous electrical stimulation has given torque-velocity profiles with an eccentric torque plateau 1.4 times the isometric value. This is closer to, but still less than, the in vitro tetanic profiles with plateaus between 1.5 and 1.9 times isometric. This study investigated the maximum voluntary and sub-maximum transcutaneous electrical stimulated torque-angle-angular velocity profiles for the knee extensors and flexors in a group of healthy males. Fifteen male subjects performed maximum voluntary and submaximum electrically stimulated ( $\sim 40 \%$ for extensors and $\sim 20 \%$ for flexors) eccentric and concentric knee extension and flexions on an isovelocity dynamometer at velocities ranging from $\pm 50^{\circ} \mathrm{s}^{-1}$ to $\pm 400^{\circ} \mathrm{s}^{-1}$. The ratio of peak eccentric to peak isometric torque $\left(T_{\text {ecc }} / T_{0}\right)$ was compared between the maximum voluntary and electrically stimulated conditions for both extensors and flexors, and between muscle groups. Under maximum voluntary conditions the peak torque ratio, $T_{\text {ecc }} / T_{0}$, remained close to $1(0.9-1.2)$ while for the electrically stimulated conditions it was significantly higher $(1.4-1.7$; $p<0.001)$ and within the range of tetanic values reported from in vitro studies. In all but one case there was no significant difference in ratios between the extensors and flexors. The results showed that even the largest muscle groups have an intrinsic $T_{\text {ecc }} / T_{0}$ comparable with in vitro muscle tests, and it can be ascertained from appropriate in vivo testing.
\end{abstract}

Keywords: $\quad$ maximum velocity, electrically stimulated, quadriceps, hamstrings 


\section{INTRODUCTION}

Maximum strength and power varies with the velocity of muscular contractions and the length of the muscle fibres. The tetanic force-velocity relationship in isolated muscle fibres follows a well established profile characterised by an eccentric force plateau at approximately $1.5-1.9$ times the isometric value, and a hyperbolic decay in force with increasing shortening velocity (Hill, 1938; Katz, 1939; Délèze, 1961; Edman et al., 1978; Edman, 1988; Harry et al., 1990). Maximum torque expressed at the joint level is a complex integration of the muscle fibre contractile properties with the in vivo architecture of multiple muscle fibres, connective tissue and neural input. In vivo measurements of maximum voluntary contraction's (MVC) forcevelocity show differences to the in vitro tetanic profile, with eccentric forces not increasing much above isometric and tending to decline with increasing lengthening velocity (Westing et al., 1988; Dudley et al., 1990; Weber and Kriellaars, 1997; Kellis and Baltzopoulos, 1998; Forrester and Pain, 2010). Consequently, maximum voluntary eccentric strength is much lower than one might expect based on maximum isometric measurements and in vitro tetanic force-velocity behaviour. EMG studies have pointed to a $10-30 \%$ reduction in the neural drive of the agonist muscle under the high loading conditions of eccentric and low concentric maximum voluntary knee extensions (Stauber, 1989; Westing et al., 1991; Kellis and Baltzopoulos, 1998; Babault et al., 2001). This is regarded to be an involuntary mechanism to protect the human body against excessive strain and injury (Westing et al., 1991).

Transcutaneous electrical stimulation to supplement maximum voluntary contractions has been found to increase eccentric knee extension torque to above the maximum voluntary levels, but to have no significant effect on concentric torque (Dudley et al., 1990; Westing et al., 1990). However, subjects are not able to tolerate the development of maximum torques through transcutaneous electrical stimulation as the sole source of knee extensor activation. Within this limitation constant stimulation levels that produced $40-60 \%$ of MVC were used by Dudley et al. (1990) to reproduce a torque-velocity profile for the knee extensors that was more similar to the in vitro tetanic profile; maximum eccentric torque was 1.4 times the isometric value and did not drop off at higher lengthening velocities.

Despite the long standing proposal of a tension limiting mechanism it is still uncertain whether neural factors are solely responsible for the difference between MVC and in vitro tetanic eccentric forces as even stimulated in vivo eccentric to isometric ratios have invariably been lower than in vitro ratios. There may be other structural components of whole in vivo muscle tendon complexes, such as changes in pennation angle with force levels (Rutherford \& Jones, 1992; Herbert \& Gandevia, 1995; Aagaard et al., 2001) or myofacial force transmission (Rijkelijkhuizen et al., 2005), that contribute. Pain and Forrester (2009) investigated correcting the maximum voluntary torque - velocity profile by using normalized, wavelet transformed EMG. They found a theoretical ratio of peak eccentric to isometric torque of 1.6 indicating that the majority, but not all, of the decreased torque, compared to in vitro could be accounted for by sub-maximal activation of the knee extensors. 
Determining to what extent the in vitro-in vivo difference is due to neural factors, and if it is consistent across muscle groups, could aid with implementing realistic eccentric muscle modelling and gaining insight for developing training and rehabilitation programmes. The aim of this study was to compare MVC and submaximum stimulation torque-angle-angular velocity profiles for the knee extensors and flexors, the first time that the knee flexors have been examined under transcutaneous electrically stimulated conditions, in a group of healthy males,. The eccentric-concentric velocity range over which the measures were taken exceeded that used in previous knee extensor studies and it is considered that this will aid in producing results commensurate with in vitro studies. It was hypothesized that the peak torque ratio (eccentric / isometric) would be higher for stimulation compared to MVC in both extensors and flexors, but that there would be no difference in the peak torque ratio between extensors and flexors as differences seen in vivo are likely predominantly due to a neural mechanism.

\section{METHODS}

Fifteen male subjects who were either university level athletes or good recreational athletes (age $23 \pm 2$ yrs, body mass $77 \pm 7 \mathrm{~kg}$, height $178 \pm 6 \mathrm{~cm}$ ) were recruited. All subjects had been injury free in their lower limbs for at least 12 months prior to testing and provided voluntary informed consent in accordance with the approval given by the University Ethical Advisory Committee.

A set protocol was completed on an isovelocity dynamometer (Con-Trex, CMV AG, Switzerland) over three sessions each separated by one week: familiarisation; knee extensors; and knee flexors. In each session subjects were seated on the dynamometer with their dominant leg strapped tightly to the unpadded crank arm directly above the ankle joint using a protective moulded plastic shin guard. To minimise differences between the crank and joint kinematics, the rotational axis of the crank arm was aligned with the centre of the knee joint during near-maximal efforts separately for both knee extension and knee flexion trials. Hip angle was controlled at $85^{\circ}$ hip flexion for the extensors and $70^{\circ}$ hip flexion for the flexors allowing subjects to exert maximal effort over as wide a knee angle range as possible with minimum discomfort, especially during stimulation trials. An initial gravity correction trial was performed, which involved the relaxed leg being moved through the full range of motion.

The protocol for the main test sessions included MVC and sub-maximum stimulation (where the transcutaneous stimulation was the only source of activation) isometric, concentric and eccentric knee extensions / flexions. Following a warm up, maximum voluntary isometric torque was measured at five angles equally distributed across the subject's range of motion. Maximum voluntary eccentric-concentric trials were measured at 10 angular velocities $\left( \pm 100,200,300,400,50^{\circ} \mathrm{s}^{-1}\right)$ following the protocol developed by Yeadon et al. (2006) with two repetitions at each velocity and a rest interval of at least two minutes between each trial. Knee range of motion was from $5^{\circ}$ to $100^{\circ}$ of knee flexion for the quadriceps and $5^{\circ}$ to $90^{\circ}$ for the hamstrings $\left(0^{\circ}\right.$ corresponded to an extended knee). This process was repeated for the stimulation trials. Finally, a single MVC isometric trial at an intermediate angle was repeated to test for fatigue effects. 
Transcutaneous electrical stimulation of the quadriceps and hamstrings was achieved using a stimulator (DS7AH, Digitimer Ltd., UK) controlled by Spike 2 software (CED micro 1401, CED, Cambridge, UK) that produced square wave impulse trains of single pulse duration $100 \mu \mathrm{s}$ at $50 \mathrm{~Hz}$. Two carbon-rubber electrodes (140 mm × $100 \mathrm{~mm}$; Electro-Medical Supplies, Greenham, UK) were coated with a thin layer of conductive gel and then taped over the rectus femoris, vastus medialis and vastus lateralis, or the biceps femoris and semi-tendinosus. To familiarise the subject with the sensation, stimulation began at a current of $40 \mathrm{~mA}$ and increased in steps of $10-30 \mathrm{~mA}$ until the prescribed level of torque was achieved. Stimulation level was calculated based on a percentage of maximal voluntary isometric torque at the middle of the five angles, $\sim 40 \%$ for the extensors, and $\sim 20 \%$ for the flexors. To limit fatigue and discomfort, each isometric trial involved stimulation for no more than one second, while in the eccentric-concentric isovelocity trials the first repetition was passive and the second stimulated. Torque and stimulation data were available in real-time to check the percentage stimulation based on voltage output and fatigue. To determine accurate joint angles and angular velocities the maximum voluntary isovelocity trials at $200^{\circ} \mathrm{s}^{-1}$ and $400^{\circ} \mathrm{s}^{-1}$ for each subject were recorded with high speed video at $200 \mathrm{~Hz}$ (Phantom V4, Vision Research, Inc., Wayne, NJ) and the video images were manually digitized. Hip, knee and ankle joint centres were marked up with black ink after being determined by manual palpation and the cosine rule used to determine the knee joint angle.

Dynamometer and stimulator data were recorded simultaneously at $512 \mathrm{~Hz}$ with Spike2 software. The dynamometer data was filtered at $8 \mathrm{~Hz}$ using a low-pass 4th order zero-lag Butterworth filter and subsequently used to obtain MVC and stimulation torque-angle-angular velocity datasets for the extensors and flexors of each subject. The crank angle and angular velocity were converted to joint angle and angular velocity based on a linear regression of joint angle against crank angle obtained from the digitised video images. For each isovelocity trial, the single maximal eccentric phase and the single maximal concentric phase were identified and the isovelocity plateau defined to be where the velocity was within $10 \%$ of the peak value. The isovelocity torques were interpolated using quintic splines (Wood and Jennings, 1979) to give an equal number of values at each velocity. A smoothed estimate of the experimental dataset for each subject, condition and muscle group was evaluated by applying a physiologically based nine-parameter torque-angle-angular velocity function as described by Forrester et al. (2011) to the experimental datasets (Figure 1). The nine parameters defining maximum torque were obtained using a Simulated Annealing algorithm (Corana et al., 1987) in which the parameter values were varied within bounds, in order to minimise a weighted root mean square difference (RMSD) between the nine-parameter fitted surface and experimental torques.

The analysis focused on the comparison of the ratio of peak eccentric torque to peak isometric torque $\left(\mathrm{T}_{\mathrm{ECC}} / \mathrm{T}_{0}\right)$ and the differential activation, characterized by $\mathrm{a}_{\mathrm{MIN}}$, between conditions and muscle groups. The torque ratio, $\mathrm{T}_{\mathrm{ECC}} / \mathrm{T}_{0}$, was evaluated based on three methods: (i) RAW: from the raw experimental data single peak values from any velocity for $\mathrm{T}_{\mathrm{ECC}}$ and $\mathrm{T}_{0}$; (ii) FITTED-1: from the nine-parameter surface fit values for $T_{E C C}$ and $T_{0}$; (iii) FITTED-2: from the nine-parameter surface fit value for $\mathrm{T}_{\mathrm{ECC}}$, and the three-parameter isometric torque-angle fit for $\mathrm{T}_{0}$. Two fitting 
methods were used as there is potential for the isometric MVC to result in a low velocity eccentric due to movement of the subject relative to the crank arm. The values of the torque ratio and differential activation were compared using a two-way ANOVA with Bonferroni post-hoc analysis where significance was set at $p<0.05$.

a

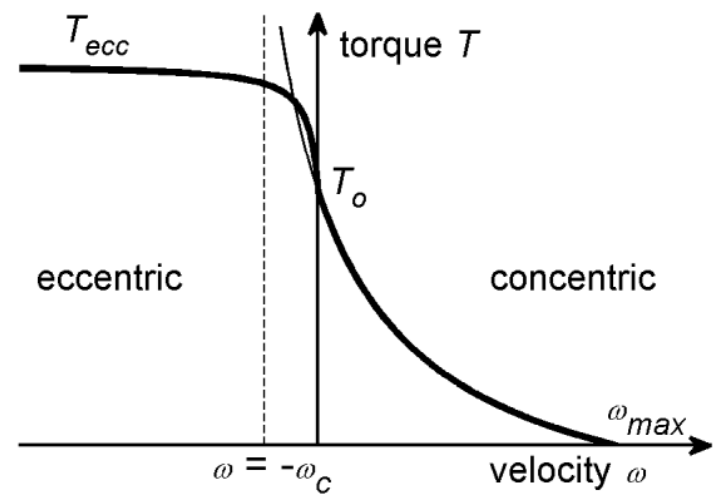

b

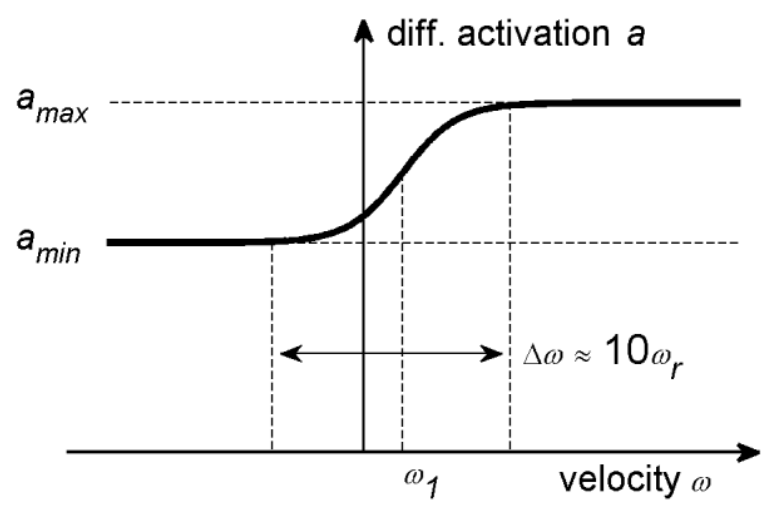

C

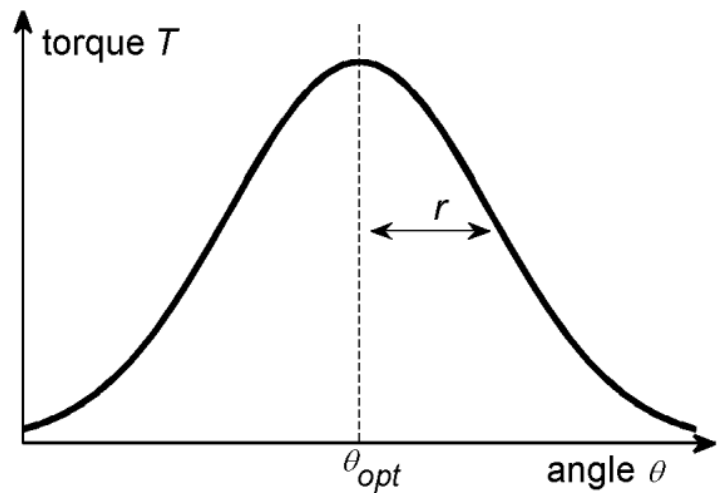

Figure 1. Components of the nine-parameter function (Forrester et al., 2011). (a) Tetanic torque angular velocity function, comprising a Hill-type hyperbola in the concentric phase and an inverted rectangular hyperbola in the eccentric phase. $k$ is the ratio of slopes between the concentric and eccentric phases and is set to a value of 4.3 representing the theoretical value predicted by Huxley's (1957) original model. The four parameters are: maximum eccentric torque $\left(T_{\text {ecc }}\right)$; maximum isometric torque $\left(T_{o}\right)$; maximum angular velocity $\left(\omega_{\max }\right)$; and angular velocity defining the vertical asymptote of the concentric hyperbola $\left(\omega=-\omega_{c}\right)$. (b) Differential activation - angular velocity sigmoid ramp up function. The three parameters are: the low plateau activation level $\left(a_{\min }\right) ; \omega_{r}$ which gives the angular velocity range over which the ramp occurs $\left(\sim 10 \omega_{r}\right)$; and the midpoint angular velocity of the ramp $\left(\omega_{1}\right)$. (c) Torque - angle function described by a normal distribution function. The two parameters are: width (standard deviation) of the curve $(r)$; and optimal angle (mean) for torque production $\left(\theta_{\text {opt }}\right)$. 


\section{RESULTS}

Adjusting the crank kinematics based on the high speed video data gave angle differences (crank versus joint) of up to $20^{\circ}$, indicating the importance of determining joint level datasets in dynamometer testing. There were no major fatigue effects, retest torques $<5 \%$ mean drop, largest single drop 9\%. Electrically stimulated torque level for the extensors was $41 \pm 6 \%$ and for the flexors $22 \pm 13 \%$ of MVC. The weighted RMSD between the experimental data and the FITTED surfaces for the extensors and flexors was $10-12 \%$ and $12-22 \%$ of the maximum isometric torque obtained in each condition.

There were significant main effects for activation condition on $T_{\text {ecc }} / T_{0}$ and differential activation $\left(a_{\text {MIN }}\right)($ all $p<0.001)$ for all methods, and one significant interaction effect between condition and muscle for raw $(p<0.005)$. $T_{\text {ecc }} T_{0}$ was significantly greater for the stimulation condition $(1.54-1.79)$ compared to the MVC $(0.93-1.19)$ (Table 1). Similarly $a_{\text {MIN }}$ was significantly greater for the stimulation condition $(0.88-0.92)$ compared to the MVC $(0.76-0.80)$ (Table 1$)$. However, when comparing the extensors and flexors the only significant difference occurred in the raw value of $\mathrm{T}_{\mathrm{ecc}} / \mathrm{T}_{0}$ under stimulation conditions (Table 1).

Table 1. Group mean $\pm S D$ for the ratio of peak eccentric torque to peak isometric torque and differential activation for the flexors and extensors, MVC and stimulation. Both the raw experimental data and surface fitted results are given.

* indicates where significant differences were observed between the MVC and stimulation ratios $\mathrm{P}<0.001$. $\S$ indicates where significant differences were observed between the extensors and flexors.

\begin{tabular}{|c|c|c|c|c|}
\hline & \multicolumn{2}{|c|}{ Knee extensors } & \multicolumn{2}{|c|}{ Knee flexors } \\
\hline & MVC & Stimulation & MVC & Stimulation \\
\hline $\mathrm{T}_{\mathrm{ecc}} / \mathrm{T}_{0}(-)$ & & & & \\
\hline${ }^{1}$ RAW & $0.93 \pm 0.12$ & $1.79^{\star} \pm 0.40$ & $1.00 \pm 0.14$ & $1.44^{\star \S} \pm 0.32$ \\
\hline${ }^{2}$ FITTED-1 & $1.17 \pm 0.05$ & $1.73^{\star} \pm 0.17$ & $1.19 \pm 0.07$ & $1.70^{\star} \pm 0.22$ \\
\hline${ }^{3}$ FITTED-2 & $1.00 \pm 0.05$ & $1.54^{\star} \pm 0.22$ & $1.02 \pm 0.05$ & $1.71^{*} \pm 0.33$ \\
\hline${ }^{4} a_{\min }(-)$ & $0.804 \pm 0.066$ & $0.921^{*} \pm 0.064$ & $0.764 \pm 0.067$ & $0.877^{*} \pm 0.093$ \\
\hline
\end{tabular}

${ }^{1}$ RAW was evaluated as the ratio of peak torque measured during the eccentric trials to the peak torque measured in the isometric trials

${ }^{2}$ FITTED-1 was evaluated as the ratio of the peak eccentric torque to peak isometric torque from the torque-angle-angular velocity surface fit.

${ }^{3}$ FITTED-2 was evaluated as the ratio of the peak eccentric torque from the torque-angle-angular velocity surface fit to peak isometric torque from the torque-angle fit to only the isometric data.

${ }^{4}$ Fitted differential activation represents the lower level of the sigmoid ramp curve and the fraction by which the tetanic torques are lowered to obtain the maximum voluntary torques in the eccentric and low velocity concentric region (Forrester et al., 2011; Yeadon et al., 2006)

A summary of the raw peak torque data achieved by subjects for knee extensors and flexors is presented in Table 2. Raw peak torque occurred at much higher velocities for the stimulation condition (Table 2). For the MVC trials peak eccentric torque occurred at either $-50^{\circ} \mathrm{s}^{-1}$ or $-100^{\circ} \mathrm{s}^{-1}$ in 23 of the 30 datasets and then dropped of rapidly (Figure 2). For the stimulation trials there was a more even distribution of peak eccentric torque occurrence across velocities over the range $-100^{\circ} \mathrm{s}^{-1}$ to $400^{\circ} \mathrm{s}^{-1}$, and generally the eccentric torques plateaued or continued increasing with increasing eccentric velocity (Figure 2 ). The raw torque-angle-angular velocity data for stimulation tended to follow the in vitro tetanic pattern much more closely than for MVC (Figure 3). The raw data were also noticeably noisier for the flexors compared to the extensors under both MVC and stimulation conditions. 
Table 2. Mean $\pm S D$ of the raw torque data for the extensors and flexors, MVC and stimulation. The velocity represents the eccentric velocity at which the peak eccentric torque occurred.

\begin{tabular}{cccccc}
\hline & \multicolumn{2}{c}{ Knee extensors } & \multicolumn{2}{c}{ Knee } & flexors \\
& & MVC & Stimulation & MVC & Stimulation \\
\hline $\mathrm{T}_{0}{ }^{\mathrm{PK}}$ & $(\mathrm{Nm})$ & $261 \pm 59$ & $101 \pm 19$ & $141 \pm 40$ & $25 \pm 11$ \\
& $\left(\mathrm{Nm} \mathrm{BW}^{-1}\right)$ & $0.344 \pm 0.063$ & $0.135 \pm 0.035$ & $0.185 \pm 0.042$ & $0.033 \pm 0.014$ \\
\hline $\mathrm{T}_{\mathrm{ECC}}{ }^{\mathrm{PK}}$ & $\left(\mathrm{Nm}^{2}\right)$ & $242 \pm 56$ & $152 \pm 28$ & $138 \pm 30$ & $42 \pm 13$ \\
\hline \multirow{2}{*}{$\begin{array}{c}\text { Velocity } \\
\text { for } \mathrm{T}_{\mathrm{ECC}}\end{array}$} & $\left({ }^{\circ} \mathrm{s}^{-1}\right)$ & $-123 \pm 78$ & $-273 \pm 139$ & $-87 \pm 52$ & $-217 \pm 136$ \\
\hline
\end{tabular}

\section{MVC}

Stimulation

Knee extensors
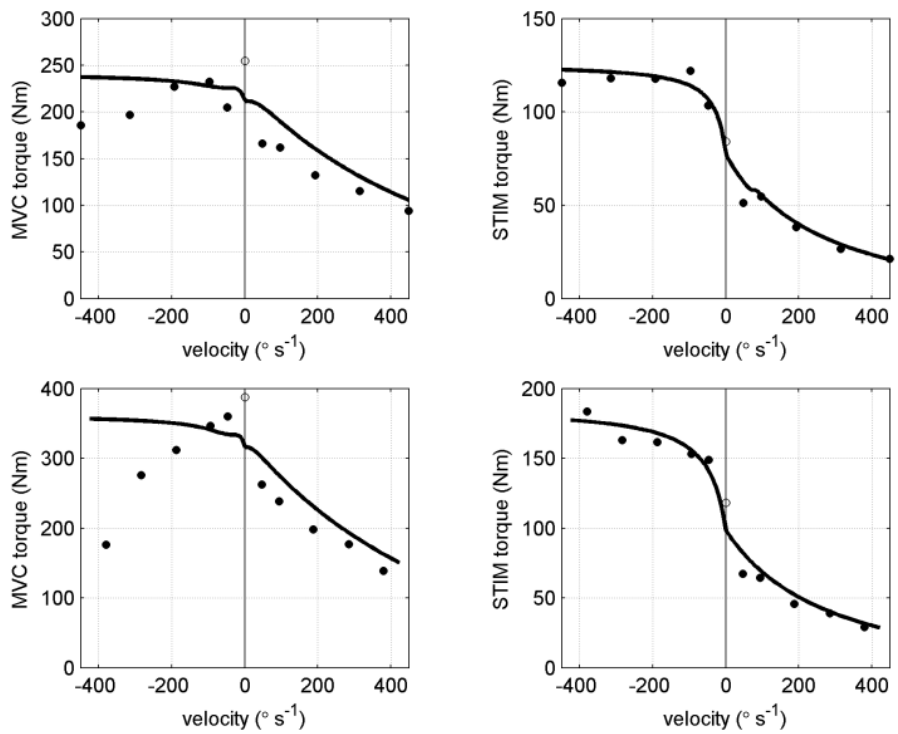

Knee flexors
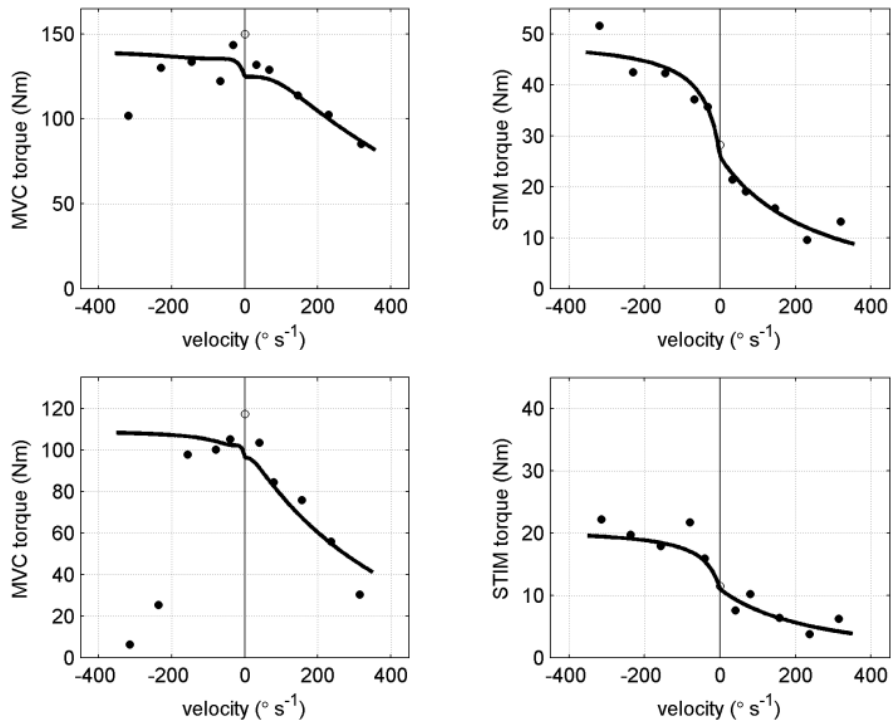

Figure 2. Typical raw peak torque - velocity data for four different subjects demonstrating the range of eccentric responses measured. The left column is for MVC and the right column is for stimulation, the first two rows are the extensors and the second two rows are the flexors. 


\section{Knee extensors}
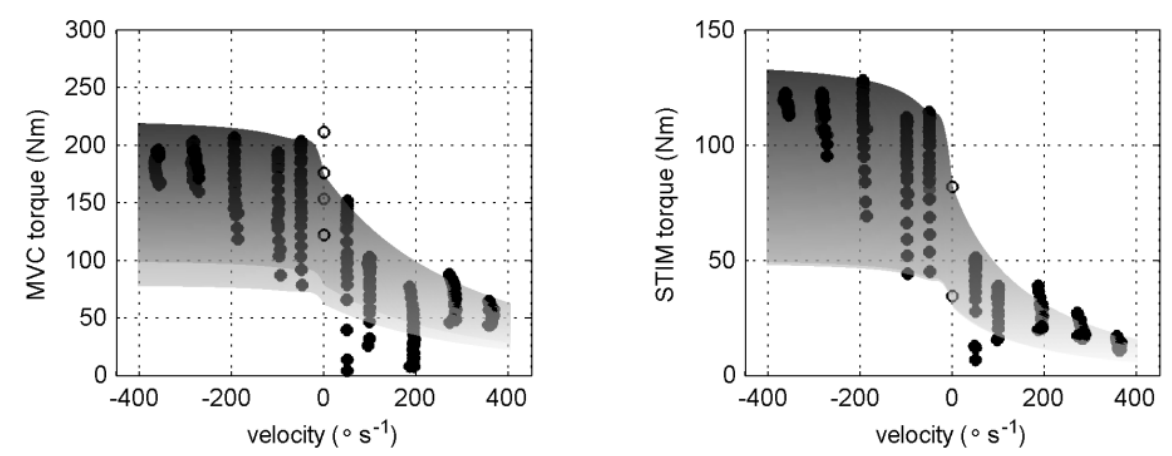

Knee flexors
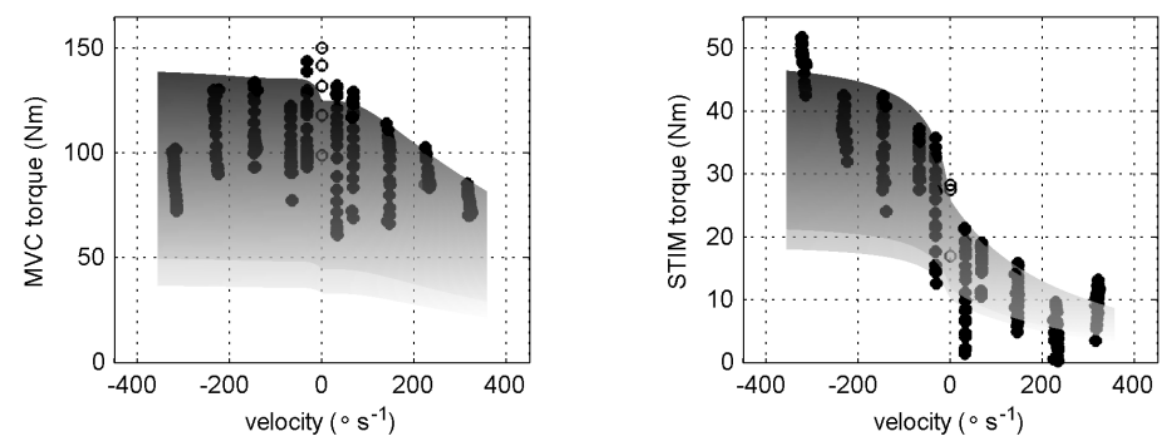

Figure 3. Typical torque-angle-angular velocity raw data (black circles) and fitted surfaces for the extensors of one subject (top row) and flexors of another subject (bottom row). The left column is for MVC and right column is for stimulation.

\section{DISCUSSION}

This study aimed to compare MVC and sub-maximum stimulation torque-angleangular velocity profiles for the knee extensors and flexors in a group of healthy males. For both muscle groups, the $T_{\text {ecc }} / T_{0}$ ratio was found to be significantly greater for stimulation compared to MVC $(1.44-1.79$ versus $0.93-1.19 ; \mathrm{p}<0.001)$. The only significant difference between muscle groups was for the raw data under stimulation conditions, and may have been a consequence of the subjects struggling with the discomfort associated with even low levels of electrical stimulation for the hamstrings. Thus, the results broadly supported the original hypotheses.

As in earlier studies for the quadriceps (Westing and Seger, 1989; Dudley et al., 1990; Westing et al., 1990; Webber and Kriellaars, 1997; Kellis and Baltzopoulos, 1998; Seger and Thorstensson, 2000) and hamstrings (Westing and Seger, 1989; Kellis and Baltzopoulos, 1998) MVC eccentric torques did not rise much, if at all, above isometric torques and dropped off with increasing velocity. At the higher velocities, where torque had not been previously measured, the drop off became more rapid and resulted in the peak MVC eccentric torque generally being recorded at $-50^{\circ} \mathrm{s}^{-1}$ or $-100^{\circ} \mathrm{s}^{-1}$. In contrast, the stimulated eccentric torques approximately 
plateaued with increasing velocity with the peak torque recorded at velocities from $100^{\circ} \mathrm{s}^{-1}$ upwards.

The results for the $\mathrm{T}_{\text {ecc }} / \mathrm{T}_{0}$ ratios reflect the raw torque-angle-angular velocity datasets, where overall, the stimulation trials clearly follow the in vitro tetanic pattern much more closely than the MVC trials. For the stimulation condition $T_{\text {ecc }} / T_{0}$ ranged between 1.4 - 1.8. The lower end of this range is similar to values reported in previous in vivo stimulation studies for the quadriceps (Dudley et al., 1990; Westing et al., 1991) soleus (Pinniger et al., 2000) and adductor pollicis (De Ruiter et al., 2000; Lee and Herzog, 2002). While the upper end is similar to values found in previous in vivo stimulation studies for the first dorsal interosseus (Cook and McDonagh, 1995) and in vitro studies for mouse and frog muscle fibres (Katz, 1939; Délèze, 1961; Edmann, 1988; Lombardi and Piazzesi, 1990; Krylow and Sandercock, 1997).

Given the level of variability in the raw data (see standard deviations in Table 2) it was considered that the fitted methods gave a more consistent and reliable set of results with regard to determining the torque ratio $T_{\text {ecd }} / T_{0}$. For the stimulated extensors, a $T_{\text {ecc }} / T_{0}$ of $1.73 \pm 0.17$ was slightly higher than the EMG corrected value found in Pain and Forrester (2009) for the same muscle group. The fitted ratio values for stimulated flexors and extensors of $1.5-1.7$ were higher than the value of 1.4 reported by Dudley et al. (1990) and may be due to the inclusion of measured torque at higher eccentric velocities and / or the fitting procedure giving an extrapolated eccentric maximum value. Indeed, the fitted ratio values in this study fall more in line with the measured in vitro tetanic values reported in the literature of 1.6 - 2.0 (Katz, 1939; Délèze, 1961; Edmann, 1988; Harry et al., 1990; Lombardi and Piazzesi, 1990; Krylow and Sandercock, 1997).

The quadriceps and hamstrings are both large muscle groups but they are different in terms of musculo-tendinous architecture, size, strength (here the quadriceps were about $80 \%$ stronger than the hamstrings) and function, with the quadriceps predominantly acting as single joint muscles and the hamstrings predominantly as two joint muscles. Despite these differences they both tended to the same $T_{\text {ecd }} / T_{0}$ ratio comparable to those seen during in vitro studies. Given the mechanical differences between the muscles and the similar outcome for $T_{\text {ecc }} / T_{0}$ it would appear that factors such as changes in pennation angle or myofacial force transmission are negligible, at least at these levels of stimulation. However, these mechanical factors may be more prominent at maximal loads which as yet have not been tested under stimulated conditions. As many of the interactions at the neural level were bypassed with the use of electrical stimulation, by staying at lower levels of stimulation and away from extreme ranges of motion, the muscle actions produced during submaximum stimulation may represent the uninhibited capacity of the muscle at that level of activation (Westing et al., 1991). This would make it likely that similar results would be found from other muscle groups under equivalent stimulated conditions as the basic muscle physiology is expressed when the neural inhibition is removed.

Limitations of this study include the lower levels of transcutaneous electrical stimulation applied to the hamstrings compared to the quadriceps and the typically noisy experimental data which is likely a result of the discomfort associated with these measurements. Using the physiological surface model helped to overcome 
the noise, however, obtaining reliable stimulation data for the hamstrings remains a challenging area. Also, the torque ratio based on the FITTED-1 method (peak isometric from the nine-parameter fit) tended to be higher, in all but the flexor stimulation condition, than that from the FITTED-2 method (peak isometric torque from the three parameter fit to the isometric data). This results from the experimental isometric torque measurements tending to be greater than the dynamic measurements (Figures 2 and 3 ) which is a common observation in dynamometer testing of the knee extensors, (Forrester et al., 2011) possibly due to the isometric joint measurements involving a slow eccentric action due to the femur being pushed forward as the pelvis and torso stabilize.

This is the first time that results from sub-maximum transcutaneous electrical stimulation of the flexors has been presented for torque-angle-angular velocity surfaces and a very similar outcome to that of the extensors was found. These results indicate that even the largest muscle groups have an intrinsic $T_{\text {ecd }} T_{0}$ comparable with in vitro muscle tests, and that it can be ascertained from appropriate in vivo testing. 


\section{REFERENCES}

Aagaard, P., Simonsen, E.B., Andersen, J.L., Magnusson, S.P., Halkjaer-Kristensen, J., Dyhre-Poulsen, P., 2000. Neural inhibition during maximal eccentric and concentric quadriceps contraction: effects of resistance training. Journal of Applied Physiology 89, 2249-2257.

Babault, N., Pousson, M., Ballay, Y., van Hoecke J., 2001. Activation of human quadriceps femoris during isometric, concentric and eccentric contractions. Journal of Applied Physiology 91, 2628-2634.

Cook, C.S., Mcdonagh, M.J.N., 1995. Force Responses to Controlled Stretches of Muscle-Tendon Complex. Experimental Physiology 80, 477-490.

Corana A, Marchesi M, Martini C and Ridella S, 1987, Minimizing multimodal functions of continuous variables with the "Simulated Annealing" algorithm. ACM Transactions on Mathematical Software, 13(3), 262-280.

Délèze, J.B., 1961. The mechanical properties of the semitendinosus muscle at lengths greater than its length in the body. Journal of Physiology 158, 154164.

De Ruiter, C.J., Didden, W.J.M., Jones, D.A., Haan, A.D., 2000. The force-velocity relationship of human adductor pollicis muscle during stretch and the effects of fatigue. Journal of Physiology 526, 671-681.

Dudley, G.A., Harris, R.T., Duvoisin, M.R., Hather, B.M., Buchanan, P. 1990. Effect of voluntary vs. artificial activation on the relationship of muscle torque to speed. Journal of Applied Physiology 69, 2215-2221.

Edman, K.A.P., Elzinga, G., Noble, M.I.M., 1978. Enhancement of mechanical performance by stretch during tetanic contractions of vertebrate skeletal muscle fibres. Journal of Physiology 281, 139-155.

Edman, K.A.P., 1988. Double-Hyperbolic Force-Velocity Relation in Frog Muscle Fibres. Journal of Physiology 404, 301-321.

Forrester, S.E., Pain, M.T.G., 2010. A combined muscle model and wavelet approach to interpreting the EMG signals from maximal dynamic knee extensions. Journal of Applied Biomechanics 26, 62-72.

Forrester, S.E., Yeadon, M.R., King, M.A., Pain, M.T.G., 2011. Comparing different approaches for determining joint torque parameters from isovelocity dynamometer measurements. Journal of Biomechanics 44, 955-961.

Harry J.D., Ward A.W., Heglund N.C., Morgan DL, McMahon TA, 1990. Crossbridge cycling theories cannot explain high-speed lengthening behaviour in frog muscle. Biophysical Journal 57, 201-208.

Herbert, R.D., Gandevia, S.C., 1995. Changes in pennation with joint angle and muscle torque: in vivo measurements in human brachialis muscle. Journal of Physiology 484, 523-532.

Hill, A.V., 1938. The heat of shortening and the dynamic constants of muscle. Proceedings of the Royal Society of London. Series B 126, 135-142.

Huxley, A.F., 1957. Muscle structure and theories of contraction. Progress in Biophysics and Biophysical Chemistry 7, 225-318.

Katz, B., 1939. The relation between force and speed in muscular contraction. Journal of Physiology 96, 45-64.

Kellis, E., Baltzopoulos, V., 1998. Muscle activation differences between eccentric and concentric isokinetic exercise. Medicine and Science in Sports and Exercise 30, 1616-1623. 
Krylow, A.M., Sandercock, T.G., 1997. Dynamic Force Responses of Muscle Involving Eccentric Contraction. Journal of Biomechanics 30, 27-33.

Lee, H.D., Herzog, W., 2002. Force enhancement following muscle stretch of electrically stimulated and voluntarily activated human adductor pollicis. Journal of Physiology 545, 321-330.

Lombardi, V., Piazzesi, G., 1990. The Contractile Response During Steady Lengthening of Stimulated Frog Muscle Fibres. Journal of Physiology 431, 141171.

Pain, M.T.G., Forrester, S.E., 2009. Predicting maximum eccentric strength from surface EMG measurements. Journal of Biomechanics 42,1598-1603.

Perrine J.J., Edgerton V.R., 1978. Muscle force-velocity and power-velocity relationships under isokinetic loading. Medicine and Science in Sports 10, 159-166.

Pinniger, G.J., Steele, J.R., Thorstensson, A., Cresswell, A.G., 2000. Tension regulation during lengthening and shortening actions of the human soleus muscle. European Journal of Applied Physiology 81, 375-383.

Rijkelijkhuizen, J.M., Baan, G.C., de Haan, A., de Ruiter, C.J., Huijing, P.A., 2005. Extramuscular myofascial force transmission for in situ rat medial gastrocnemius and plantaris muscles in progressive stages of dissection. Journal of Experimental Biology 208, 129-140.

Rutherford, O.M., Jones, D.A., 1992. Measurement of fibre pennation using ultrasound in the human quadriceps in vivo. European Journal of Applied Physiology 65, 433-437.

Seger, J.Y., Thorstensson,A., 2000. Electrically evoked eccentric and concentric torque-velocity relationships in human knee extensor muscles. Acta Physiologica Scandinavica 169, 63-69.

Stauber W.T., 1989. Eccentric action of muscles: physiology, injury, and adaptation. Exercise and Sport Science Reviews 17, 157-185.

Weber, S., Kriellaars, D., 1997. Neuromuscular factors contributing to in vivo eccentric moment generation. Journal of Applied Physiology 83, 40-45.

Westing, S.H., Cresswell, A.G., Thorstensson, A., 1991. Muscle activation during maximal voluntary eccentric and concentric knee extension. European Journal of Applied Physiology 62, 104-108.

Westing, S.H., Seger, J.Y., Thorstensson, A., 1990. Effects of electrical stimulation on eccentric and concentric torque-velocity relationships during knee extension in man. Acta Physiologica Scandinavica 140, 17-22.

Westing, S.H., Seger, J.Y., Karlson, E., Ekblom, B., 1988. Eccentric and concentric torque-velocity characteristics of the quadriceps femoris in man. European Journal of Applied Physiology 58, 100-104.

Wickiewicz, T.L., Roy, R.R., Powell, P.L., Perrine, J.J., Edgerton, V.R., 1984. Muscle architecture and force-velocity relationship in humans. Journal of Applied Physiology 57, 435-443.

Wood, G.A., Jennings, L.S., 1979. On the use of spline functions in data smoothing. Journal of Biomechanics 12, 477-479.

Yeadon, M.R., King, M.A., Wilson, C., 2006. Modelling the maximum voluntary joint torque / angular velocity relationship in human movement. Journal of Biomechanics 39, 476-482. 